International Journal of Ophthalmology \& Eye Science (IJOES)

ISSN 2332-290X

\title{
Health Coaching and Glaucoma: A Qualitative Evaluation of the Patient Experience in a Preliminary Study
}

Jullia A. Rosdahl ${ }^{1 *}$, Suzanne Schneider ${ }^{2}$, Anita P. Vin ${ }^{1}$

${ }^{1}$ Department of Ophthalmology, Duke University, Durham, North Carolina, USA.

${ }^{2}$ Department of Health Philosophy and Promotion, Maryland University of Integrative Health, Laurel, Maryland, USA.

\begin{abstract}
Introduction: Successful medical treatment of glaucoma requires adherence to daily eye drop medications. Health coaching, which has been used successfully for other chronic diseases, may be helpful for glaucoma patients.

Methods: Qualitative analysis of pre-coaching questionnaire, coaching notes, and post-coaching exit interviews, for four glaucoma patients who completed a 6-month health coaching interventional study. The health coaching intervention consisted of 4-6 coaching sessions with a certified Health Coach via telephone.

Results: Preserving independence was the most important theme identified prior to coaching. Participants varied in focus areas, focusing on either eye health, general health, or a combination. The health coaching intervention resulted in benefits for all four patients, from the development of eye drop adherence strategies to weight loss and work-life balance.

Conclusion: These results support the use of holistic approaches to care for glaucoma patients, as well as further research on the use of health coaching for glaucoma patients and the development of health coaching interventions tailored to the unique needs of glaucoma patients.
\end{abstract}

Keywords: Glaucoma; Health Coach; Coaching; Medication Adherence; Barriers to Adherence; Qualitative Research.

\section{*Corresponding Author:}

Jullia A. Rosdahl MD PhD,

Department of Ophthalmology, Duke University, Durham, North Carolina 27710, USA.

E-mail: jullia.rosdahl@duke.edu

Received: June 24, 2015

Accepted: July 10, 2015

Published: July 14, 2015

Citation: Jullia A. Rosdahl, Suzanne Schneider, Anita P. Vin (2015) Health Coaching and Glaucoma: A Qualitative Evaluation of the Patient Experience in a Preliminary Study. Int J Ophthalmol Eye Res. S1:007, 32-35. doi: http://dx.doi.org/10.19070/2332-290X-SI01007

Copyright: Jullia A. Rosdahl ${ }^{\circ}$ 2015. This is an open-access article distributed under the terms of the Creative Commons Attribution License, which permits unrestricted use, distribution and reproduction in any medium, provided the original author and source are credited.

\section{Introduction}

Glaucoma is a group of diseases that if left untreated can cause blindness, and it is currently managed by lowering eye pressure. The most common eye pressure lowering treatments are eye drop medications [1] that require instillation at least daily. A patient's adherence to the prescribed medication regimen is required for successful treatment of this chronic disease, but adherence is complex and often inadequate [2]. Strategies that have been successful for other chronic diseases may be helpful for glaucoma $[3,4]$.

Health coaching can help in the management of chronic diseases such as diabetes and cardiovascular disease [5], where daily use of chronic medications is needed and where the early disease is usually asymptomatic, similar to glaucoma. Health coaches use openended directed conversations to support positive behavior change based upon where the individual is in the stages of change. Health coaching is based on the fundamental idea that the individual is the best source for solutions to their own health challenges [6]. One of the techniques used by health coaches, motivational interviewing, has been shown to be feasible in ophthalmology clinics [7].

In this exploratory study, we sought to qualitatively investigate the use of health coaching for glaucoma patients. The health coaching intervention required that the coach touch on eye health during each session while allowing the patient to focus on areas of their choice. Since health coaching itself is open-ended, this approach left open the possibility that unanticipated aspects of health coaching could be helpful for glaucoma patients.

\section{Methods}

\section{Study participants}

Glaucoma patients were recruited for participation in a prospective pilot study to use Health Coaching in the care of glaucoma. The study protocol was approved by the Duke University Institutional Review Board; study participants were in the study for a total of six months, starting with an initial visit when demographic information was collected, followed by the health coaching intervention, ending with an exit interview. Four glaucoma patients completed the pilot study. 


\section{Health coaching intervention}

The health coaching intervention consisted of four to six sessions of health coaching with a certified health coach, based on the Duke Integrative Health Coaching model [8]. In an introductory session (approximately one hour) the coach reviewed the coaching process, discussed the patient's assessment of various domains of health and wellbeing, and started to explore the impact of disease and treatment. The subsequent sessions lasted approximately forty-five minutes, every two to four weeks. Health coaches use open-ended questions, reflective listening techniques, and guided discussions to explore participants' vision concerning eye and general health, the values that motivate change, and their barriers to behavior change. All of the coaching sessions were carried out via telephone.

\section{Data collection}

This qualitative study draws from three data sources:

1. Participants completed a Personal Health Plan (PHP) when they first enrolled in the study. Adapted from Duke Integrative Medicine's Integrative Health Coach Professional Training Program, the PHP focused on five primary areas: 1 . View of overall optimal health; 2 . View of optimal vision health; 3. Personal health assessment relating to eight areas of self-care and healthcare; 4. Ranking of health assessment areas; and 5. Goals of coaching.

2. Coaching notes were completed by the study coach to document details of each coaching session. Coaching notes focused on six areas: 1. Focus of session; 2. Strengths identified; 3. Barriers identified; 4. Key insights; 5. Action steps; and 6 . Time distribution (eye health vs. general health)

3. Semi-structured exit interviews were completed with each study participant after completion of their coaching sessions. The exit interview guide focused on three key areas: 1.The health coaching experience; 2 . Health coaching and glaucoma; and 3. The study experience. All interviews were audiorecorded, transcribed, and de-identified.

\section{Data analysis}

Content analysis of PHPs, coaching notes, and exit interviews were conducted to:

1. Develop comprehensive profiles of individual participants, their health coaching experience, and their behavior changes. Data charts for each data source were developed for building and analyzing participant profiles.

2. Generate patterns and themes from aggregate data. Participant data were coded and sorted by code to identify key patterns and themes that emerged across cases.

We examined participant profiles and aggregate data themes to elicit trends regarding eye drop barriers, health concerns, and coaching efficacy.

\section{Results}

\section{Vision and values in glaucoma patients}

The glaucoma patients who participated in this coaching study were similar in age and race to our glaucoma population (age 6983 years; race: black/white, 2/2; gender: male/female, 3/1). Prior to the coaching sessions, all of the participants completed a questionnaire, the PHP, about their values and their perspectives on the future. Interestingly, all participants identified "independence" as most important on an open-ended query about their vision of optimal health: as one participant stated, "as I age, (wanting to) be in a well state and living on my own.” Maintaining a healthy weight, having energy, and looking good were also identified as components of optimal health and to be "able to approach each day with vigor, energy, flexibility, independence."

The descriptions of optimal visual health varied considerably among the participants, from being able to do correspondence and reading, to regaining vision, to driving confidently at night. The global importance of vision was summarized by one participant: "being able to work and drive and read and travel." All participants recognized the risk of vision loss in the future from glaucoma, with a likely scenario of "maintaining fair vision" and a worst case scenario of "blindness."

Eye care was rated as the number one area of health by all participants. Mindful awareness, as well as movement and exercise, were also identified as high priority areas of health.

\section{The health coaching experience}

The participants completed four to six coaching sessions, including an introductory session and a closing session. The health coaching experience was assessed from both the coach's perspective using coaching notes and from the participants' perspectives using exit interviews.

The health coach touched on eye health during each of the coaching sessions, but the focus of each session was directed by the participant. One participant chose to focus all of his sessions on eye health. Another focused almost solely on general health. The other 2 participants divided the time between eye health and general health.

The participant who focused predominantly on eye health was struggling with the mid-day drop for his thrice-daily-dosed glaucoma medication. His coaching focused on improving his adherence, and through the coaching, he developed his own solutions including a wood block bottle holder and an electronic spreadsheet to record his adherence. At his closing session, the "lessons learned were: 1 . There can be flexibility in drop spacing; 2. Pills and drops are different; 3. Every day is different in retirement which makes eye drop routine difficult."

In his exit interview after the coaching, he noted that glaucoma had great personal significance to him because he watched his mother lose her sight from the disease. At the outset he was committed to working through the "mid-day eye drop problem". He identified the key barrier as his own belief about the exact spacing of the drops, and through the coaching and conversations with his doctor, became more flexible. His impression of health coaching was ambiguous: he noted that it was "too broad" and became "too personal" and that it "needed to be more health-focused and wellness-oriented" and "emphasize being able to see," but that there were beneficial aspects: 
Through the coaching and through the feedback that I've been getting here it was to relax and do it when it was convenient, not become overly concerned about the precise interval between eye drops.

He appears to have derived benefit for his glaucoma care through the health coaching, but the coaching process was not entirely comfortable for him.

The participant who focused primarily on general health noted during the introductory session that felt he was "doing a great job with eye health" and that he wanted to focus on weight loss; he identified a key barrier as "little down time/too much work." His subsequent coaching sessions focused on weight loss, including mindful eating, and work-life balance. For work-life balance, he focused on decreasing work time, prioritizing self-time and decreasing obligations, and through the coaching, was able to decrease his workload and increase his personal time, noting "good progress" in this area when reviewing his accomplishments in the final coaching session.

In his exit interview, he noted that "coaching made me think about what I was doing" and helped him to "finally decide to do something about the weight control." There were several specific changes that he made due to the coaching:

I have been more compliant with my diet. I go now to see an internist that I haven't seen for years. I was going to the ophthalmologist and a hematologist at one time but I haven't had any general physical examination and since the coaching started I have been going to an internist at least twice and I have been followed more frequently so that was good.

For one, it made me change the way and basically just a little bit taking more time off from work. For example, now I'm taking some weekends and I haven't taken a weekend in years...The other thing is just make me go to a doctor regularly. Those were very positive changes I thought.

He reported that the coaching "helped me become more aware of the problem (eye drops) but I was pretty aware of the problem before" and he suggested that non-adherent patients might benefit most from health coaching.

Two of the participants focused on eye health and general health topics. For example, one of the participants focused on diet and exercise for the first three sessions, including changes in eating and exercise, and on the eye drops and her difficulty with the daytime drop for the remaining session. In her exit interview, she noted her long-standing commitment to her eye health, with over 40 years of medication use and keeping appointments, and also cited a family member's experience with glaucoma: her "brother lost his eyesight, but was diagnosed much later than she was." She reported that the "coaching helped [her] get focused on things she hadn't thought about before, to identify and strategize how to correct things" and that she had "made changes in eating healthier on account of coaching." Similar to another participant, she said that "new, younger patients just diagnosed with glaucoma would benefit most from coaching." She described her coaching experience:
It was fun. It really got me actually to identify some things I really hadn't thought about. I just hadn't given some of the things that we did discuss, I hadn't thought about at all. I don't go around thinking about what would happen if I lose my eyesight. I don't think about it. It helped me to realize that maybe there were some things I that I wanted to do that I needed to do.

Even though she was an experienced glaucoma patient, the coaching helped her to gain additional perspective on her situation and increased her self-awareness.

\section{Health coaching benefits}

The health coaching intervention offered support for behavior change to help improve eye health and general health. Benefits of the health coaching were seen in eye health and general health. With regards to eye health, participants developed specific strategies to improve adherence as well as enhanced self-awareness about their vision and disease. With regards to general health, the benefits included weight loss and diet changes, more exercise, adjustments to work-life balance with more family time, and reconnection to primary care.

\section{Benefits for glaucoma eye drops:}

Well, I've become more rigorous in trying to know when, I think it has probably sharpened my time awareness as to how I want to take these drops and the fact that I'm recording it and now it is kind of a game, so it's good. Actually I now try a little harder than otherwise to make sure I don't miss.

\section{Benefits for general health:}

It made me think about what I was doing. I finally decided I was going to do something about the weight control. I have done it, it's not completed but I lost a significant amount of weight. I was going to take more time off and force the issue that I needed more time off and carve the time to be able to take time off. It has resulted in a more balanced lifestyle. Basically I have more time for my family. She did not coach me into doing anything... but basically she was making me see what was going on and made me make some decisions about issues which are important for health care. For example, to have enough rest, to control my weight.

\section{Benefits for vision:}

The most valuable to me was identifying the areas that I had not thought about before. Basically just hadn't given them any though. Like how would losing my sight affect my activities of daily living? How would losing sight affect my overall being? Would I be able to drive and go when I wanted to or just do things when I wanted to do them without the assistance of someone else?

\section{Discussion}

In this qualitative study of glaucoma patients who participated in health coaching, the intervention was flexible allowing partici- 
pants to focus on eye health and/or general health areas. The benefits of health coaching extended from eye drop adherence strategies to weight loss and work-life balance.

The most important theme for participants at the start of the study was preserving independence. Maintaining good vision is important for the maintenance of independence into older adulthood although significant progress has been made in Vision Rehabilitation [9]. While remaining independent was important to all of the participants when they considered their aspirations for optimal health at the start of the coaching, it was only a focus area for one of the participants during the coaching, in identifying low vision resources, and was not identified as a theme in the exit interviews. The participants did however note how coaching had helped bring about a wider perspective, to "see what was going on and ... make some decisions about issues which are important for health care" and to "identify some things I had not thought about before."

Retirement and work is another area of interest in the glaucoma population, with many glaucoma patients of retirement age and some with visual disability affecting work. Two of the participants who were retired and still quite active struggled with daytime drop instillation; one noted the "complexity of life and schedules. Traveling and time changes made tracking drops difficult." One of the participants in this study was still very actively working, and the health coaching benefited him in the area of general health. Though a small sample size, this study reveals the diverse situa- tions and needs of glaucoma patients.

Older patients especially those with visual impairment frequently have multiple medical problems [10] and all of our study participants had at least one other chronic medical problem requiring medication besides glaucoma. Health coaching has the potential to affect other aspects of health [6], in addition to glaucoma, for the patients in the study. The health coaching intervention used in this study was open-ended, allowing participants to direct the coaching focus, and showed benefits both in eye health and general health. This study supports the use of a holistic approach to caring for patients with glaucoma, as patients with multiple medical problems including glaucoma.

There are limitations to this study. A small number of glaucoma patients participated in the study, thus the results may not be generalizable, and all of the participants received coaching (no control group). The study duration was six months and persistent effects from coaching were not assessed. The open format of qualitative studies limits the ability to measure the quantitative effects of coaching. Effects on medication adherence are not presented in this qualitative manuscript. Effects on physiology such as actual weight loss were not measured. The effects of the health coaching were assessed using a comprehensive evaluation based on pre-coaching values exercise (PHP questionnaire), coaching notes (taken during the coaching), and participant recall (during the exit interview).

Table 1. Pearls For Patient Care.

\begin{tabular}{|c|}
\hline Pearls for caring for the glaucoma patient \\
\hline Glaucoma patients have diverse barriers, and diverse solutions to overcome them. \\
\hline An essential tenant of health coaching is that patients have their own solutions. \\
\hline Consider training a staff member as a coach to help patients with adherence issues. \\
\hline Health coaching can benefit older patients in unexpected ways. \\
\hline
\end{tabular}

The glaucoma patients in this study derived benefits in various ways from health coaching, from improved adherence strategies for glaucoma drops to enhanced work-life balance; highlights are summarized in Table 1. These results support the use of holistic approaches to care for glaucoma patients, as well as further research on the use of health coaching for glaucoma patients and the development of health coaching interventions tailored to the unique needs of glaucoma patients.

\section{Acknowledgements}

This work was funded by a grant from the American Glaucoma Society.

\section{References}

[1]. Vass C, Hirn C, Sycha T, Findl O, Bauer P, Schmetterer L (2007) Medi$\mathrm{cal}$ interventions for primary open angle glaucoma and ocular hypertension. Cochrane Database Syst Rev 2007(4): CD003167.

[2]. Tsai JC (2009) A comprehensive perspective on patient adherence to topical glaucoma therapy. Ophthalmology 116(11 Suppl): S30-36.

[3]. Waterman H, Evans JR, Gray TA, Henson D, Harper R (2013) Interventions for improving adherence to ocular hypotensive therapy. Cochrane Database Syst Rev 2013(4): CD006132.

[4]. Viswanathan M, Golin CE, Jones CD, Ashok M, Blalock SJ, et al. (2012) Interventions to improve adherence to self-administered medications for chronic diseases in the United States: a systematic review. Ann Intern Med
157(11):785-795.

[5]. Wolever RQ, Webber DM, Meunier JP, Greeson JM, Lausier ER, et al. (2011) Modifiable disease risk, readiness to change, and psychosocial functioning improve with integrative medicine immersion model. Altern Ther Health Med 17(4):38-47.

[6]. Moore M (2013) Coaching the Multiplicity of Mind: A Strengths-based Model. Glob Adv Health Med 2(4):78-84.

[7]. Cook PF, Bremer RW, Ayala AJ, Kahook MY (2010) Feasibility of motivational interviewing delivered by a glaucoma educator to improve medication adherence. Clin Ophthalmol 4:1091-1101.

[8]. Wolever RQ, Caldwell KL, Wakefield JP, Little KJ, Gresko J, et al. (2011) Integrative health coaching: an organizational case study. Explore (NY) 7(1):30-36.

[9]. Liu CJ, Brost MA, Horton VE, Kenyon SB, Mears KE (2013) Occupational therapy interventions to improve performance of daily activities at home for older adults with low vision: a systematic review. Am J Occup Ther 67(3): 279-287.

[10]. Court H, McLean G, Guthrie B, Mercer SW, Smith DJ (2014) Visual impairment is associated with physical and mental comorbidities in older adults: a cross-sectional study. BMC Med 12:181.

Special Issue on

"Glaucoma and Hypertensive Retinopathy"

Edited by:

Dr. Pinakin Gunvant Davey, Western University of Health Sciences, USA

E-mail: pdavey@westernu.edu 